\begin{tabular}{|c|c|}
\hline UÁQUIRI & PPG \\
\hline Heve & Revista do Programa de Pós-Graduação em Geografia \\
Home page: https://periodicos.ufac.br/index.php/Uaquiri & ISSN 2675-4088 \\
\hline
\end{tabular}

\title{
(Relatos de Experiências) \\ POTENCIALIDADES E DESAFIOS DOS MAPEAMENTOS COLABORATIVOS VIRTUAIS NO CONTEXTO DE PANDEMIA
}

\author{
Gabriel Brandão Xavier ${ }^{*}$, Guilherme Ignácio Reis ${ }^{2}$, Iasmin Castro ${ }^{1}$, Fábio da Silva Lucio ${ }^{1}$, \\ Fernanda Lima-Silva ${ }^{3}$, Lívia Castro Degrossi ${ }^{3}$ \\ ORCID: https://orcid.org/0000-0001-8071-1862; https://orcid.org/0000-0001-8573-4633; \\ https://orcid.org/0000-0002-5617-1656; https://orcid.org/0000-0002-7273-7648; \\ https://orcid.org/0000-0003-4838-7075; http://orcid.org/0000-0001-6897-1186;
}

${ }^{1}$ Universidade Federal do Acre, Centro de Filosofia e Ciências Humanas, Rio Branco, Acre, Brasil. ${ }^{2}$ Universidade Federal Fluminense, Instituto de Ciências da Sociedade e Desenvolvimento Regional, Campo dos Goytacazes, Rio de Janeiro, Brasil. ${ }^{3}$ Centro de Estudos em Administração Pública e Governo da Fundação Getúlio Vargas Escola de Administração de Empresas de São Paulo (CEAPG/FGV EAESP), São Paulo, Brasil.

*gabrielbx188@gmail.com

Recebido em: 26/05/2020 Aceito em: 23/09/2020 Publicado em: 20/12/2020 DOI: https://doi.org/10.47418/uaquiri.vol2.n2.2020.3744

\begin{abstract}
RESUMO
Recentemente, os mapeamentos colaborativos se tornaram uma peça fundamental na produção e democratização do acesso a dados geográficos. Mapas com dados abertos, editáveis e acessíveis por qualquer pessoa podem ser uma importante ferramenta para apoiar ações relacionadas ao território. $\mathrm{O}$ objetivo deste artigo é apresentar as potencialidades e os desafios dos mapeamentos colaborativos feitos presencialmente e compará-los com mapeamentos colaborativos realizados inteiramente de forma virtual, devido ao isolamento social provocado pela pandemia de Covid-19. Relatou-se uma experiência de ensino e extensão composta por capacitação e mutirões de mapeamento colaborativo com o OpenStreetMap para mapear o município de Rio Branco - Acre. Como resultado dos mutirões virtuais de mapeamentos colaborativos, os voluntários adicionaram novos objetos geográficos ao mapa digital, oferecendo um mapa base para ações como decisões logísticas ou socorristas antes, durante ou depois a pandemia. Por outro lado, atestou-se que a infraestrutura tecnológica como acesso à internet e familiaridade com a tecnologia da informação ainda representam um grande desafio aos voluntários.
\end{abstract}

Palavras-chave: Mapeamento colaborativo; OpenStreetMap; Pandemia.

\section{POTENTIALS AND CHALLENGES OF VIRTUAL COLLABORATIVE MAPPING IN THE PANDEMIC CONTEXT}

\begin{abstract}
Recently, collaborative mapping has become a fundamental part in the production and democratization of the access to geographic data. Maps with open, editable and accessible data can be an important tool to support actions related to a territory. The purpose of this article is to present the potentials and challenges of collaborative mapping activities made in-person and compare them with collaborative
\end{abstract}


mapping activities carried out entirely in a virtual form, due to the social isolation caused by the pandemic of Covid-19. We report teaching and extended learning experiences composed of training and collaborative mapping efforts with OpenStreetMap to map the municipality of Rio Branco - Acre. As a result of virtual collaborative mapping efforts, volunteers added new geographic objects to the map, offering a base map for actions as logistical decisions or first responders before, during or after the pandemic. On the other hand, we verified that technological infrastructure as internet connection and familiarity with information technology still represent a great challenge for volunteers.

Keywords: Collaborative Mapping; OpenStreetMap; Pandemic

\section{POTENCIALIDAD Y DESAFÍOS DEL MAPEO COLABORATIVO VIRTUAL EN EL CONTEXTO PANDÉMICO}

\section{RESUMEN}

Recientemente, el mapeo colaborativo se ha convertido en una parte fundamental en la producción y democratización del acceso a datos geográficos. Los mapas con datos abiertos, editables y accesibles por cualquier persona pueden ser una herramienta importante para apoyar acciones relacionadas con el territorio. El propósito de este artículo es presentar los potenciales y desafíos de los mapeos colaborativos realizados en persona y compararlos con los mapeos colaborativos realizados completamente en forma virtual, debido al aislamiento social causado por la pandemia de Covid-19. Se informó que una experiencia de enseñanza y extensión que consiste en capacitación y esfuerzos de mapeo colaborativos con OpenStreetMap para mapear el municipio de Rio Branco - Acre. Como resultado de los esfuerzos de mapeo colaborativo virtual, los voluntarios agregaron nuevos objetos geográficos al mapa digital, ofreciendo un mapa base para acciones tales como decisiones logísticas o primeros respondedores antes, durante o después de la pandemia. Por otro lado, se atestiguó que la infraestructura tecnológica como el acceso a Internet y la familiaridad con la tecnología de la información aún representan un gran desafío para los voluntarios.

Palabras-clave: Mapeo colaborativo; OpenStreetMap; Pandemia.

\section{INTRODUÇÃO}

O mapeamento colaborativo pode ser entendido como uma atividade colaborativa para a geração de dados sobre as características do espaço geográfico, isto é, dados geográficos sobre ruas, rodovias, edificações, entre outros. Um benefício do mapeamento colaborativo é, por exemplo, a geração de uma grande quantidade de dados geográficos em um curto período de tempo sobre as áreas afetadas por um desastre natural (ZOOK et al., 2010; POIANI, et al., 2016). A atividade de mapeamento colaborativo pode ser dividida em capacitação, que visa descrever como os elementos do espaço geográfico são mapeados, e mutirão de mapeamento, onde os indivíduos mapeiam de fato os elementos. Tradicionalmente, ambas atividades são realizadas pelos indivíduos de forma presencial. Contudo, a pandemia ocasionada pelo Covid$19^{1}$ impôs o isolamento social e impossibilitou a condução das atividades presencialmente. Este

\footnotetext{
${ }^{1}$ Coronavírus da síndrome respiratória aguda grave 2 (SARS-CoV-2).
} 
cenário abriu novas oportunidades para que as práticas de mapeamentos colaborativos fossem repensadas frente à nova realidade.

Este trabalho relata a experiência de ensino e extensão composta por capacitação e mutirões de mapeamento colaborativo realizados inteiramente de forma virtual. O objetivo deste relato é apresentar as potencialidades e os desafios das atividades de mapeamento colaborativo realizadas presencialmente e compará-los com as atividades de mapeamento colaborativo realizadas inteiramente de forma virtual devido ao isolamento social ocasionado pela pandemia. As atividades de mapeamento colaborativo visaram mapear o município de Rio Branco (Acre) e foram realizadas por meio de uma parceria entre alunos e pesquisadores da Universidade Federal do Acre (UFAC) e pesquisadores do projeto Dados à Prova D’água (em inglês, Waterproofing Data), que desenvolvem atividades relacionadas à governança dos riscos de desastres.

Durante as atividades, alunos, pesquisadores e voluntários utilizaram a plataforma de mapeamento colaborativo OpenStreetMap ${ }^{1}$ (OSM), cujo objetivo é criar um banco de dados geográficos de todo o mundo e disponibilizá-lo na forma de mapa digital gratuito e editável (HAKLAY; WEBER, 2008). As plataformas de mapeamento colaborativo, como o OSM, são importantes ferramentas pois permitem que indivíduos espalhados ao redor do mundo sejam capazes de mapear remotamente uma área de interesse, auxiliando na geração de dados geográficos.

Um diferencial do OSM é que ele permite que os mapas sejam produzidos e modificados por técnicos e, também, pela população que vive e conhece a área de interesse. Por isso, o OSM é visto como uma ferramenta que promove o empoderamento e a democratização do acesso e da produção de dados. Propor e realizar uma capacitação e mutirões de mapeamento em formato online e gratuito também são meios de democratização, considerando que ações como essa ampliam o poder de entendimento e intervenção da sociedade civil. Discute-se, nesse processo, o conceito de empoderamento estrutural (KLEBA; WENDAUSEN, 2009), que é a viabilização da corresponsabilização e participação social na perspectiva do engajamento cidadão.

O mapeamento feito pela comunidade pode ser usado para complementar as lacunas existentes nos mapas oficiais e aprimorar mapas feitos por técnicos, que muitas vezes não conhecem a área de fato. Além disso, mapear colaborativamente possibilita a geração de dados

\footnotetext{
${ }^{1}$ https://www.openstreetmap.org/
} 
geográficos que podem ser usados para as mais diversas ações, sejam elas ambientais, assistenciais ou em saúde, além de ser um modo de transformar as condições que afetam diretamente a qualidade de vida da população.

O restante deste documento está estruturado da seguinte forma: Na Seção 2 são descritos o método tradicional de mapeamento colaborativo e o mapeamento colaborativo virtual; $\mathrm{Na}$ Seção 3 são descritos os resultados e as lições aprendidas enquanto na Seção 4 as considerações finais são apresentadas, os entendimentos sobre as maratonas de mapeamento são relatados e são apresentadas recomendações sobre a realização das atividades de mapeamento colaborativo virtual.

\section{COMPARANDO OS MÉTODOS TRADICIONAL E VIRTUAL}

A plataforma OSM foi criada em 2004 com o objetivo de constituir uma base de dados gratuita com dados geográficos do mundo todo e construída de maneira coletiva. Inicialmente focada em mapear ruas e estradas, a plataforma recentemente passou a ser utilizada, por exemplo, para o mapeamento de áreas afetadas por desastres naturais (POIANI et al, 2016).

Atualmente, o OSM já ultrapassa o número de 6 milhões de usuários ${ }^{1}$. O crescimento exponencial da plataforma pode ser entendido a partir da demanda pela geração de dados geográficos abertos, precisos e atualizados, e também pela facilidade no seu uso. Usuários podem começar a mapear logo após se registrarem na plataforma, adicionando ou editando objetos que representam características do espaço geográfico como ruas, prédios, pontos de interesse à base de dados da plataforma (NEIS \& ZIPF, 2012). Em situações onde um grande número de voluntários mapeia a mesma área de interesse, a ferramenta HOT Tasking Manager ${ }^{2}$, ferramenta complementar ao OSM, é utilizada para organizar os processos de mapeamento colaborativo.

Os pesquisadores do projeto Dados à Prova D’água desenvolveram uma metodologia para a realização de atividades de mapeamento colaborativo, capacitação e mutirões de mapeamento, utilizando as ferramentas OSM e HOT Tasking Manager, conforme apresentada na Figura 1. As atividades de mapeamento colaborativo, conduzidas no âmbito do projeto, têm os objetivos de disseminar o OSM no Brasil e contribuir para a melhoria do mapa da plataforma, em particular de áreas urbanas e assentamentos informais, ainda pouco mapeados. Planejadas

\footnotetext{
1 Informação extraída do site OpenStreetMap Statistics em 23/05/2020. Para mais informações, acesse https://www.openstreetmap.org/stats/data_stats.html

2 https://tasks.hotosm.org/
} 
inicialmente para um público em idade escolar, entre junho de 2019 e fevereiro de 2020 foram desenvolvidas de forma presencial com estudantes do Ensino Médio em uma escola pública em São Paulo e outra em Rio Branco e reaplicado com moradores do distrito de M'Boi Mirim (São Paulo).

Figura 1 - Estrutura de mapeamento colaborativo tradicional

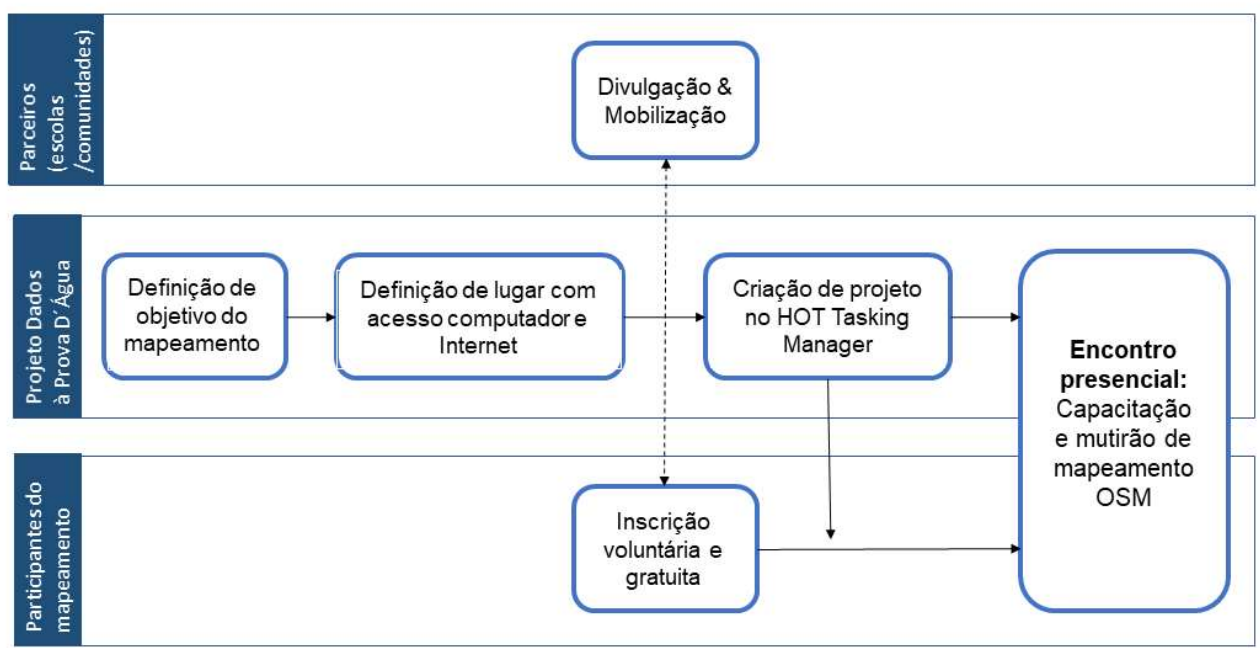

A capacitação desenvolvida nos encontros presenciais abordava a plataforma OSM e suas principais funcionalidades e era seguida pelo mutirão de mapeamento. Os coordenadores da atividade, que conduziam as atividades com o grupo, eram apoiados por uma pequena equipe de monitores, que iam de computador em computador verificando se os participantes estavam conseguindo acompanhar a capacitação. Se alguém tinha dúvidas ou dificuldades com o computador ou a plataforma, os monitores apoiavam a sua resolução. No espaço onde eram conduzidas as capacitações buscava-se criar um clima descontraído com músicas, bate-papos e cafés.

Com o cenário de isolamento social imposto pela pandemia da Covid-19, foi necessário pensar em novas maneiras de organizar e realizar atividades de mapeamento colaborativo inteiramente virtuais. As atividades de mapeamento colaborativo ocorreram nos dias 12, 19, 26 de maio e 6 de junho de 2020, e foram realizados na plataforma Zoom Meetings ${ }^{1}$. Cada uma delas durou cerca de duas horas. Sua dinâmica de realização foi diferente da efetuada no mapeamento tradicional, como pode ser observado na Figura 2.

\footnotetext{
${ }^{1}$ https://zoom.us/
} 
Para auxiliar os novos usuários e promover o uso das plataformas de mapeamento colaborativo, foram produzidos tutoriais em vídeo ${ }^{1}$ e em formato escrito ${ }^{2}$, que abordam as funcionalidades básicas do OSM e HOT Tasking Manager. Por meio de uma perspectiva ativa de ensino, os materiais foram enviados com antecedência aos participantes para que houvesse uma aproximação prévia com as plataformas utilizadas.

Figura 2 - Estrutura de mapeamento colaborativo virtual

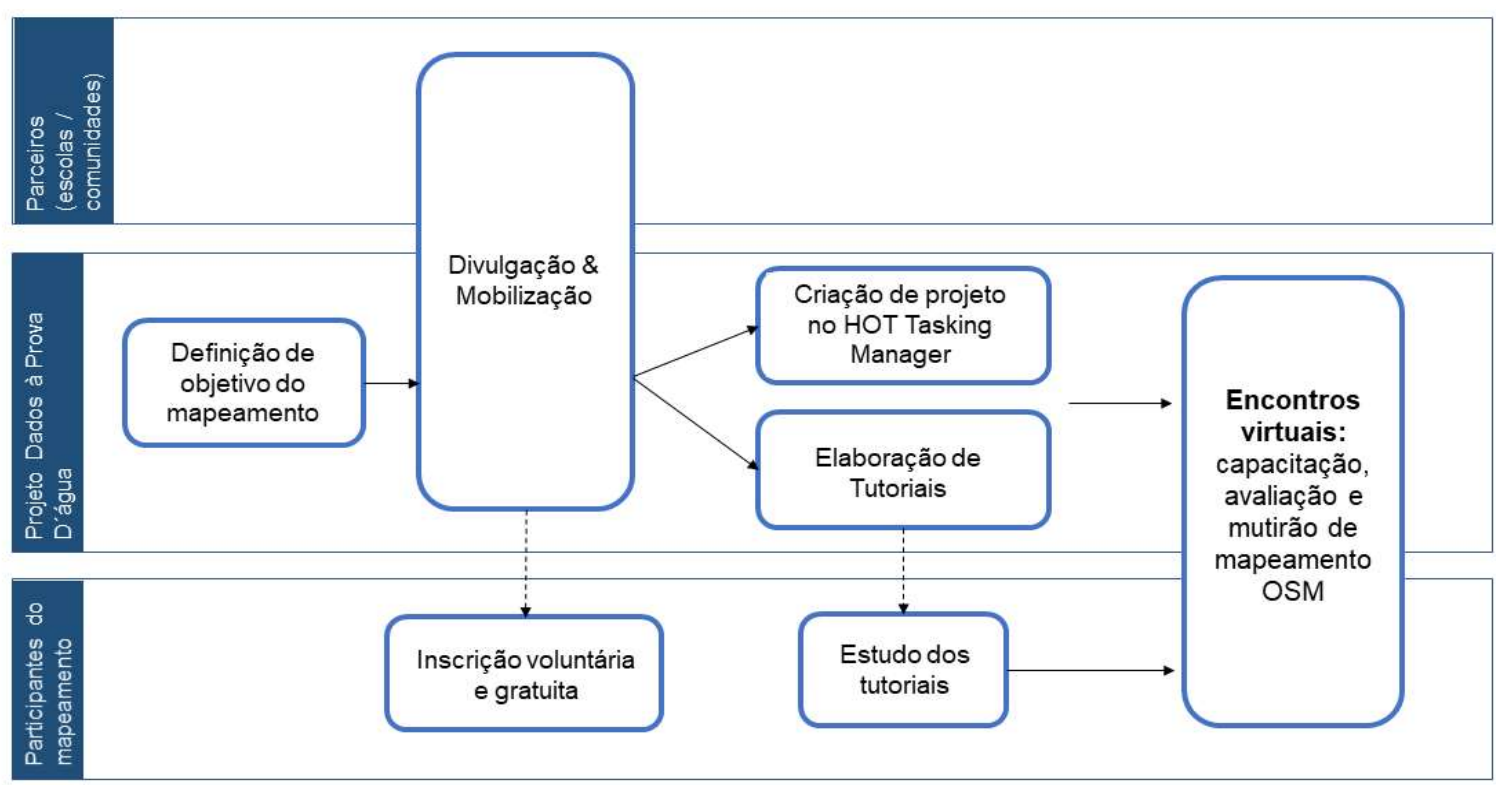

A condução do encontro virtual se baseou em três momentos. No primeiro encontro, o coordenador geral do projeto Dados à Prova D’água, Prof. Dr. João Porto de Albuquerque, discorreu sobre os aspectos centrais do mapeamento colaborativo, a plataforma OSM e sua aplicação em diferentes contextos. No segundo encontro, houve uma capacitação prática abordando as funcionalidades básicas de mapeamento no OSM e no HOT Tasking Manager, que foi ministrada por dois integrantes do projeto, enquanto outros exerceram o papel de monitores e auxiliaram os participantes com suas dúvidas, que eram escritas no chat do aplicativo de comunicação. Após estas explanações, iniciou-se o mutirão de mapeamento, que foi continuado nos últimos dois encontros.

\footnotetext{
${ }^{1}$ Link de acesso ao tutorial em vídeo: https://www.youtube.com/watch?v=Jm518M989j4\&t=3s.

2 Link de acesso ao tutorial escrito: https://ceapg.fgv.br/sites/ceapg.fgv.br/files/u60/tutorial_para_o_mapeamento_colaborativo_com_o_openstreetm ap.pdf
} 
Os mutirões de mapeamento foram realizados na plataforma HOT Tasking Manager. Nesta plataforma foi criado o projeto de mapeamento colaborativo \#6124 Mapping flood prone urban areas in Brazil ${ }^{1}$ para mapear uma região do município de Rio Branco (Acre), que é área de estudo do projeto. Todo o mapeamento feito pelos voluntários durante os mutirões incidiu sobre esse limite territorial.

\section{RESULTADOS E LIÇÕES APRENDIDAS}

O mutirão de mapeamento colaborativo realizado totalmente virtual, proposto e realizado em momento de emergência sanitária mundial, se mostrou uma ferramenta importante para dar continuidade às atividades de pesquisa, colocando a equipe em posição de repensar as antigas atividades e propor novas.

Após o início das atividades de mapeamento, pode-se observar que dentre as dificuldades encontradas pelos colaboradores, a primeira foi a ausência do recebimento dos emails contendo as instruções, tutoriais, data e demais informativos. Mesmo após verificar a caixa de spam, houve relatos do não recebimento do material. Como forma de solucionar esse problema, buscamos reenviar as informações a todos identificados, bem como criar um grupo via WhatsApp ${ }^{2}$, para que houvesse um outro meio para notificar os participantes.

As novas práticas foram bem recebidas pelos voluntários, ocasionando um bom engajamento em todas as atividades propostas. No total, 91 pessoas se inscreveram no mutirão, sendo 66 inscritos residentes do município de Rio Branco. Os demais inscritos eram residentes de municípios dos estados de São Paulo, Acre, Minas Gerais, Amazonas, Paraíba, Rio de Janeiro, Alagoas e Rondônia, conforme apresentado na Tabela 1. Os encontros contaram com a presença de 30 participantes, em média. $\mathrm{O}$ número total de participantes por encontro pode ser observado na Tabela 2 .

A realização da capacitação em um encontro específico foi positiva, uma vez que os voluntários eram mapeadores iniciantes e não tinham conhecimento sobre mapeamento colaborativo. As apresentações teóricas e práticas se complementaram e possibilitaram uma boa introdução ao tema e às funcionalidades das plataformas OSM e HOT Tasking Manager. O envio antecipado dos tutoriais contribuiu para que a maioria dos participantes tivesse um

\footnotetext{
${ }^{1}$ https://tasks.hotosm.org/projects/6124/tasks/

2 www.whatsapp.com
} 
contato prévio com o material e os ajudou a ter um melhor aprendizado durante a atividade de capacitação, conforme relatos dos participantes.

Tabela 1: Distribuição dos inscritos por local de residência

\begin{tabular}{lc}
\hline Estado & Número de Participantes \\
\hline Acre & 69 \\
São Paulo & 11 \\
Amazonas & 3 \\
Minas Gerais & 3 \\
Rio de Janeiro & 2 \\
Alagoas & 1 \\
Paraíba & 1 \\
Rondônia & 1 \\
\hline
\end{tabular}

Tabela 2: Número de participantes por encontro

\begin{tabular}{lc}
\hline Data do encontro & Número de Participantes \\
\hline 12 de maio & 37 \\
19 de maio & 36 \\
26 de maio & 25 \\
2 de junho & 24 \\
\hline
\end{tabular}

Como principais resultados dos mutirões de mapeamento, novos edifícios, áreas residenciais, áreas verdes, ruas, praças e outros elementos foram mapeados a partir da sua visualização em imagens de satélites (Figura 3). Até o momento de escrita deste relato, foram realizadas 8.147 novas edições no mapa.

Todos os voluntários contribuíram com novas edições no mapa, mesmo aqueles que não eram residentes de Rio Branco ou que residiam no município, mas não conheciam a localidade. Aqueles que tinham uma boa familiaridade com o local acrescentaram informações mais detalhadas sobre alguns elementos mapeados, como o nome de: estabelecimentos comerciais, ruas, escolas e igrejas.

Conhecendo a plataforma e como o mapeamento é realizado, os voluntários poderão seguir mapeando as regiões que conhecem, colaborando para atualização e melhoria do mapa. Centros urbanos de grandes cidades como, por exemplo, da Inglaterra são mais mapeados do que as áreas rurais e periferias (HAKLAY, 2010), devido ao maior número de pessoas que mapeiam essas regiões. Em outras palavras, cidades onde os dados são atualizados e abundantes são aquelas com maiores índices populacionais (CAMBOIM et al, 2015). As pessoas engajadas 
no processo de mapeamento do território podem, também, trazer para o mapa informações locais e atualizadas, pois estão cotidianamente naquele contexto, acompanhando as mudanças que ocorrem.

Figura 3 - OpenStreetMap (a) antes e (b) depois dos mutirões de mapeamento.

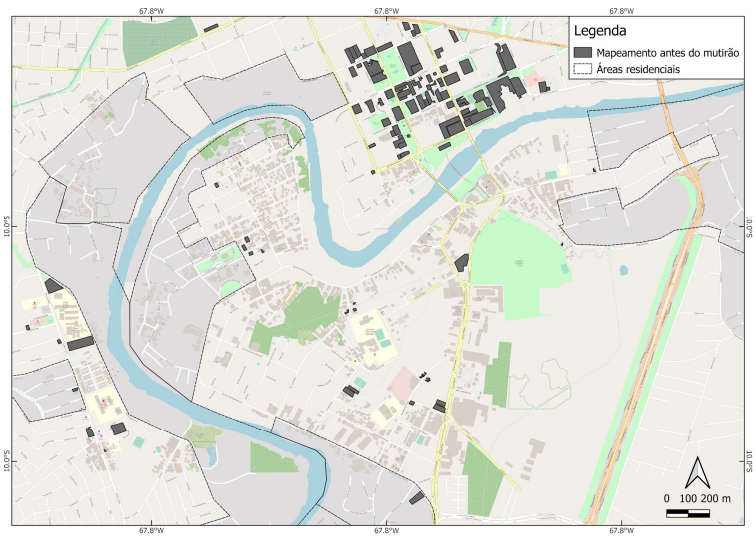

(a)

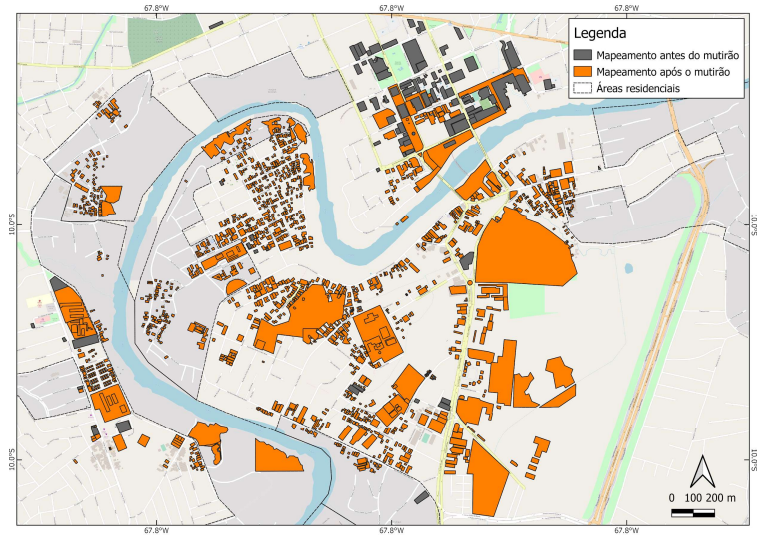

(b)

Após cada encontro, os integrantes do projeto realizaram uma avaliação da atividade e uma validação do mapeamento feito, que eram compartilhadas de forma resumida com os participantes no encontro seguinte. Neste processo, qualquer erro individual cometido por algum participante é reconhecido e corrigido, essa é uma das vantagens do mapeamento colaborativo em relação aos métodos tradicionais (ZOOK et al., 2010). Durante as validações foram encontrados alguns erros, tais como: uma mesma área mapeada 2 vezes por voluntários diferentes, uso da representação por linhas para mapear edifícios, sobreposições entre as delimitações de elementos vizinhos, dentre outros. Em uma situação presencial, os erros mencionados seriam facilmente evitados, pois haveria monitores circulando e acompanhando o que é feito em cada computador. Entretanto, esse tipo de acompanhamento se torna inviável em um ambiente inteiramente virtual.

Os monitores dependiam da interação dos participantes pelo chat para sanar qualquer dúvida, porém ela não ocorria de forma fluida e muitos participantes não faziam perguntas ou as direcionavam de forma privada para uma única monitora, gerando uma sobrecarga de trabalho. Embora algumas dúvidas tenham sido sanadas pela monitora de maneira explícita a todos os voluntários, os erros decorrentes da atividade de mapeamento só foram identificados durante as validações. Caso os mutirões de mapeamento ocorressem de forma presencial, grande parte deles poderia ser identificado e corrigido durante o andamento das maratonas. Com essas experiências, fica claro que a forma como os monitores interagem com os 
participantes deve ser repensada para os próximos eventos de mapeamento colaborativo virtuais, buscando alternativas para melhorar a comunicação entre as partes.

Durante os mutirões de mapeamento, o acesso a rede de internet se destacou como um dos principais desafios para a organização do evento. Em uma cidade como Rio Branco, geograficamente afastada dos grandes centros do país, rotineiramente as conexões de rede são instáveis ou mesmo inexistentes. O mesmo não ocorre em grandes metrópoles brasileiras, como São Paulo e Rio de Janeiro, cidades onde alguns dos inscritos nas atividades residem.

Sob esse aspecto, HAKLAY (2013) aponta a dificuldade de envolver pessoas com acesso reduzido a internet nas atividades de mapeamento colaborativo. Embora sejam mapas abertos, esta atividade ressalta a desigualdade do acesso à informação e produção de dados geográficos no Brasil. As maratonas de mapeamento virtual evidenciaram alguns casos de pessoas que não possuíam rede wi-fi em suas residências, outras que sentiam dificuldade para acompanhar os mapeamentos devido à pouca familiaridade com ferramentas computacionais. A ausência de conexão a uma rede de internet estável, por exemplo, impediu a participação de diversos interessados, que não dispõem de acesso à internet em casa no cenário atual e que, devido às restrições impostas com o isolamento social, não podem recorrer a outros meios, impossibilitando a participação.

Devido a diversidade de acessos à internet no Brasil, houve uma série de relatos de lentidão, travamentos, falha na conexão, áudio distorcido, problemas com acesso ao áudio, dentre outros. Tais problemas eram, de certo modo, esperados devido à alta latência, referente ao distanciamento do servidor, e ao elevado número de participantes. Visando contornar tais dificuldades e estabelecer um ambiente lúdico e confortável de realização das atividades, criamos estratégias para fomentar e diversificar o diálogo, como apresentações, perguntas sobre lazer, gostos pessoais, atividades realizadas na pandemia, dentre outras. A finalidade principal é que os membros possam interagir em um ambiente amigável, participativo e antiestático, tornando as maratonas um evento divertido, ao mesmo tempo que atinge seus objetivos.

\section{CONSIDERAÇÕES FINAIS}

Este relato de experiência apresentou as potencialidades e os desafios inerentes ao processo de mapeamento colaborativo em época pandêmica. Os resultados obtidos durante a experiência ratificaram a importância dos mapeamentos como forma de evolução cartográfica. Oferecer oportunidades aos indivíduos uma maneira de entender como eles se relacionam com 
o espaço geográfico e gerar novos dados aos territórios foram as principais potencialidades verificadas durante as maratonas de mapeamento colaborativo.

As maratonas de mapeamento colaborativo virtual ressaltaram ainda o potencial em manter o mapa atualizado, em comparação aos mapas oficiais e mapas disponibilizados por outras plataformas online, que podem estar desatualizados. Desse modo, as plataformas de mapeamento colaborativo oferecem um mapa base acessível e atualizados por um grande número de voluntários.

Se, por um lado, o contexto da pandemia impôs medidas restritivas aos usuários das plataformas de mapeamento colaborativo em caráter presencial; por outro, os avanços tecnológicos permitem que esses indivíduos sejam inseridos como protagonistas da sua realidade, se transformando em agentes ativos no processo de mapeamento do seu território durante a pandemia.

Em contraste às potencialidades, as dificuldades da realização das maratonas de mapeamento colaborativo de maneira totalmente virtual, conforme pontuadas anteriormente, evidenciaram a desigualdade do acesso à informação, a exclusão digital em tempos de pandemia e ressaltou as dificuldades inerentes à aprendizagem sobre as plataformas de mapeamento colaborativo. Apesar das facilidades com que dados geográficos podem ser compartilhados no OSM, a infraestrutura tecnológica, como conexão de internet e ausência de equipamentos, e a familiaridade com a tecnologia são fatores fundamentais para a inclusão digital dos indivíduos nas maratonas de mapeamento.

Diante das potencialidades e dificuldades para a realização de maratonas de mapeamento colaborativo de forma totalmente virtual em Rio Branco, recomendamos maior atenção ao feedback dos participantes como forma de aprimorar a dinâmica das maratonas e estabelecer um meio de comunicação para avisos e recomendações eficaz com os voluntários.

De maneira geral, as maratonas de mapeamento colaborativo realizadas virtualmente podem ser uma atividade enriquecedora para as comunidades, que podem entender o espaço geográfico ao qual estão inseridas. Além disso, essas comunidades colaboram para a manutenção dos mapas digitais e os tornam úteis para a vida cotidiana.

\section{AGRADECIMENTOS}

Os autores gostariam de agradecer ao laboratório de Hidrologia, Meio Ambiente e Geografia dos Riscos da Universidade Federal do Acre, à Universidade de 
Warwick, Fundação Getúlio Vargas (FGV) e a Fundação de Amparo à Pesquisa do Estado de São Paulo (FAPESP) pelo suporte nas maratonas de mapeamento virtual em Rio Branco.

\section{REFERÊNCIAS}

CAMBOIM, S. P.; BRAVO, J. V. M.; SLUTER, C. R. An investigation into the completeness of, and updates to, the Open Street Map data in a heterogeneous area in Brazil. ISPRS International Journal of Geo-Information, n.4(3), 1366- 1388. 2015.

DOI: https://doi.org/10.3390/ijgi4031366

HAKLAY, M.; WEBER, P. OpenStreetMap: User-Generated Street Maps. IEEE Pervasive Computing, v. 7 (4), p. 12-18, Oct.-Dec. 2008.

DOI: https://doi.org/10.1109/MPRV.2008.80

HAKLAY, M. How Good is volunteered geographical information? a comparative study of OpenStreetMap and ordnance survey datasets. Environment and Planning. B, Planning \& Design, 37(4), 682-703. 2010

DOI: http://dx.doi.org/10.1068/b35097

HAKLAY, M. Citizen Science and Volunteered Geographic Information: Overview and Typology of Participation. In: SUI, D.; ELWOOD S.; GOODCHILD, M. (org.) Crowdsourcing Geographic Knowledge. Springer, Dordrecht, 2013. p. 105-122.

DOI: https://doi.org/10.1007/978-94-007-4587-2 7

KLEBA, M. E,; WENDAUSEN, A. Empoderamento: processo de fortalecimento dos sujeitos nos espaços de participação social e democratização política. Saúde e Sociedade, São Paulo , v.18, n.4, p.733-743, dez. 2009 Disponível em: $<$ http://www.scielo.br/scielo.php?script=sci_arttext\&pid=S010412902009000400016\&lng=en\&nrm=iso>. Acesso em: 16 maio. 2020.

DOI: http://dx.doi.org/10.1590/S0104-12902009000400016.

NEIS, P.; ZIPF, A. Analyzing the Contributor Activity of a Volunteered Geographic Information Project - The Case of OpenStreetMap, ISPRS Int. J. Geo-Information, vol. 1, pp. 146-165, 2012.

POIANI, T. H.; ROCHA, R. d. S.; DEGROSSI, L. C.; ALBUQUERQUE, J. P. d. "Potential of Collaborative Mapping for Disaster Relief: A Case Study of OpenStreetMap in the Nepal Earthquake 2015," 2016 49th Hawaii International Conference on System Sciences (HICSS), Koloa, HI, 2016, pp. 188-197.

DOI: https://doi.org/10.1109/HICSS.2016.31

ZOOK, M.; GRAHAM, M.; SHELTON, T.; GORMAN, S. "Volunteered Geographic Information and Crowdsourcing Disaster Relief: A Case Study of the Haitian Earthquake", World Med. Heath Policy, vol. 2, no. 2, pp. 7-33, 2010.

DOI: https://doi.org/10.2202/1948-4682.1069 